\section{HÁBITAT Y ADULTO MAYOR: EL CASO DE VALPARAÍSO}

Giulietta Fadda ${ }^{1}$

Alejandra Cortés ${ }^{2}$

\section{Resumen}

Este artículo hace referencia a resultados de la última etapa (2008) de la investigación Fondecyt No 1061179, titulada "Calidad de Vida del Adulto Mayor en el Hábitat Urbano: Estudio del Caso de Valparaíso". La investigación aborda el problema de la calidad de vida de los adultos mayores en la ciudad, con particular referencia al caso de Valparaíso. Esta ciudad es un caso de estudio de especial interés, dado que tiene los mayores índices de envejecimiento de las tres grandes urbes chilenas y presenta, además, un carácter urbano-cultural y patrimonial y una escarpada configuración topográfica que alberga su heterogeneidad social.

Se exponen los resultados cuanti y cualitativos a través de indicadores de calidad de vida de este grupo etario en Valparaíso. El análisis arrojó notables diferencias en los factores que inciden en la calidad de vida de este grupo según su localización

\section{HABITAT AND SENIOR CITIZENS: VALPARAÍSO CASE STUDY}

\author{
Giulietta Fadda ${ }^{1}$
}

Alejandra Cortés ${ }^{2}$

\section{Abstract}

This article talks about the Fondecyt Research Project No. 1061179 final stage results (2008) named "Senior Citizens Quality Life in Urban Habitat: Valparaiso Case Study." The investigation addresses the problem of senior citizens quality life in the city, particularly Valparaiso. This city is a special interest case study as it has the highest ageing indexes of the three largest Chilean cities. It also depicts a urban cultural and patrimonial character and a steep topography that shelters a socially heterogeneous population.

This age group quality life indexes in Valparaiso portrays the quantitative and quality research results. The analysis carried out shows significant differences between the variables that affects this group quality life, related where they live in 
en la ciudad (cerro/plan) y su situación socioeconómica (vulnerable/estable). En coherencia a estos resultados -positivos y negativos- se hacen algunas proposiciones y sugerencias para futuras políticas públicas socio-urbanas.

\section{PALABRAS CLAVES: CALIDAD DE VIDA, ADULTO MAYOR, VALPARAÍSO.}

Fecha de Recepción: 04.04.09

Fecha de Aceptación: 23.06.09

1 Chile. Arquitecta PhD., Facultad de Arquitectura, Universidad de Valparaíso, Valparaíso.

Correo electrónico: gfadda@vtr.net

2 Chile. Arquitecta Ms., Departamento de Arquitectura, Universidad Técnica Federico Santa María, Valparaíso.

Correo electrónico: alejandra.cortes@usm.cl the city (hills/ground level) and socio-economic variable (vulnerable/stable). Some suggestions and proposal are made for future socio-urban public policy making coherent with the research results, either positive or negative ones.

\section{KEY WORDS: QUALITY OF LIFE, SENIOR ADULT,} VALPARAISO.

Received: 04.04.09

Accepted: 23.06 .09

1 Chile. Architect PhD. School of Architecture, Universidad de Valparaíso, Valparaiso.

Email: gfadda@vtr.net

2 Chile. Architect Ms., Departamento of Architecture, Universidad Técnica Federico Santa María, Valparaiso.

Email: alejandra.cortes@usm.cl 


\section{Introducción}

La ciudad de Valparaíso constituye un caso de estudio de especial interés en este tema, ya que tiene los mayores índices de envejecimiento de las tres más grandes metrópolis chilenas. Presenta, además, un exclusivo carácter urbano-cultural y patrimonial, unido a una configuración topográfica en escarpada pendiente, que alberga y segrega su heterogeneidad social. De acuerdo al último Censo (2002), mientras la tasa nacional de adultos mayores es de $11,4 \%$, en la comuna de Valparaíso llega al 13,94\%.

La investigación trata de establecer las posibles relaciones entre envejecimiento y calidad de vida en este contexto urbano. Para ello, se abordan dos ejes de análisis de la problemática de la calidad de vida urbana del adulto mayor: uno, de tipo ecológicoterritorial, donde interesan las relaciones existentes entre las condiciones espaciales de distintas zonas de Valparaíso, con respecto al bienestar, calidad de vida e integración urbana de las personas mayores; y el otro, de tipo socio-demográfico, donde se investiga la influencia de la situación socio-económica y redes sociales en que está inserto el adulto mayor.

Además, pretende avanzar en el conocimiento de las interrelaciones que se producen entre desarrollo urbano, calidad de vida y envejecimiento e indagar sobre la forma práctica en que determinadas condiciones del hábitat urbano inciden en la calidad de vida del grupo etario más anciano de la población.
Dentro de tales propósitos, los principales objetivos son: determinar dinámicas urbanas a las que están expuestos los adultos mayores; estudiar los elementos determinantes y procesos causales de una calidad de vida diferencial para los adultos mayores en la ciudad; analizar las relaciones entre envejecimiento y calidad de vida en el contexto urbano de Valparaíso y definir las grandes coordenadas de una política de intervención urbana para mejorar la calidad de vida del adulto mayor.

Para dar cumplimiento a estos objetivos, se elaboraron y compararon índices de la calidad de vida de las personasmayoresresidentesenlazona; seidentificaron relaciones entre su hábitat, calidad de vida y redes de apoyo y se jerarquizaron las condiciones del hábitat urbano que inciden, negativa o positivamente, en estos aspectos. Por último, se propusieron medidas para la adecuación de la ciudad, desde el punto de vista de la calidad de vida de las personas mayores y el fomento de sus redes de apoyo.

En el presente artículo se exponen los principales resultadosdelainvestigación. Enuna primerasección se hace referencia a la morfología de Valparaíso, como un antecedente para la integración urbana del adulto mayor. Un segundo acápite aborda la construcción de los índices, como una de las formas cuantitativas que se aplicó para medir la calidad de vida de los adultos mayores en la ciudad. Un tercero, presenta 
resultados que aúnan aspectos tanto cualitativos como cuantitativos (triangulación), especialmente referidos a indicadores que repercuten en forma más significativa sobre la calidad de vida de los adultos mayores en Valparaíso. Para su exposición, se distinguieron dos grupos: "Indicadores con incidencia negativa" e "Indicadores con incidencia positiva" y una referencia a la "Percepción General". En el último capítulo, a modo de conclusiones, se deducen los corolarios pertinentes.

\section{Morfología Urbana de Valparaíso}

La morfología urbana de Valparaíso adquiere características muy especiales, debido a su accidentada topografía, que ha representado un elemento determinante en su existencia y proceso de desarrollo. Igualmente, le ha conferido un carácter especial, ya sea en su forma física y su estructura urbana, como en su vida socio-cultural. Valparaíso es "una ciudad escindida en una zona plana ... y en una zona de relieve costero, formada por una cadena de cerros de regular altura" ${ }^{3}$, en la que el acantilado separa el plan de los cerros. La trama urbana de Valparaíso se dispone, en torno a una bahía abierta al norte, sobre una estrecha planicie costera y un "anfiteatro", conformado por

3

Waisberg, 2000 (p. 5). cuarenta y dos cerros, abierto al Océano Pacífico. Este abanico de cerros se conecta al mar por un sistema de rutas y está surcado por un régimen de quebradas convergentes al Plan ${ }^{4}$. Este hecho contribuye a darle a los cerros la condición de unidades independientes. El plan, con 5\% de la población residente y $80 \%$ de las actividades productoras de bienes y servicios, alberga el equipamiento urbano, mientras que los cerros, con 95\% de la población y alrededor del 15\% de las actividades productoras de bienes y servicios, se limitan al equipamiento vecinal.

Las características geográficas descritas reúnen condicionesquepuedenimplicarventajasodesventajas para la integración urbana de los adultos mayores. Son estos pro y contra, los que nuestra investigación pretende identificar y evaluar. Subsiguientemente, proponer medidas y políticas públicas, propiciatorias o rectificadoras, según la situación.

\section{Construcción de Índices de Calidad de Vida}

El concepto de calidad de vida 5 proporcionó la base teórico-metodológica de la investigación. Concepto que resulta de gran validez para evaluar las condiciones y bienestar de grupos sociales

4 Álvarez, 2001 (p. 2).

$5 \quad$ Fadda y Jirón, 1999 (p. 177). 
en un determinado ambiente. Esto, por cuanto estudiar la "calidad de vida" de una comunidad significa analizar no sólo las condiciones físicas y objetivas, sino las experiencias sociales y subjetivas que los individuos tienen de su existencia en su hábitat. Implica evaluar cómo viven los individuos, qué expectativas de transformación tienen tales condiciones y cuál es el grado de satisfacción que se consigue tanto a nivel individual como colectivo. En este sentido, y para cumplir con los objetivos planteados en el proyecto, se requirió recolectar información a través de instrumentos metodológicos cuantitativos y cualitativos. Además, tanto para la recolección de datos como para el análisis teórico y metodológico, se recurrió a la triangulación, es decir, a la complementación de múltiples instrumentos.

Entre los principales instrumentos cuantitativos utilizados, se cuenta la encuesta, aplicada a 480 adultos mayores. Ella se fundamentó en la información tanto cualitativa como cuantitativa recopilada anteriormente: mapas SIG, encuesta Delphi, Focus Groups en distintas zonas de Valparaíso (dos en los cerros y dos en el plan), observación en terreno y un seminario taller. Para su mejor manejo, la información recopilada se clasificó en 5 campos: humano, sociocultural, natural, físico y económico. Cada campo estuvo compuesto por múltiples indicadores y sub-indicadores de calidad de vida, los cuales correspondieron a las preguntas del cuestionario aplicado en la encuesta y a las pautas de los instrumentos cualitativos. Se definieron 21 indicadores ${ }^{6}$ de calidad de vida, focalizados al adulto mayor.

El campo humano, definido por las habilidades, conocimientos, capacidades laborales y salud ,incluyó indicadores como: uso del tiempo libre, privacidad de la vivienda, necesidades del barrio, estado de salud, nivel educacional, expectativas, apoyo afectivo y percepción de discriminación en razón de su edad.

El campo socio-cultural, relativo a redes sociales, capacidad de acción (empoderamiento), arraigo e identidad, se compuso de indicadores como: percepción del barrio, factores de inseguridad del barrio, consideración de sus opiniones, opinión respecto de sus vecinos, participación en organizaciones y apoyo que brinda a los demás.

El campo natural, concerniente a medio ambiente y sustentabilidad, abarcó indicadores como:

6 El término "indicador" implica: advertir, guiar, señalar. Da claves para entender cosas más complicadas y hacer perceptible una tendencia o fenómeno no explícito. Su objetivo es orientar la acción. Los indicadores de CV se basan simultáneamente en hechos objetivos observables de condiciones de vida y en percepciones y evaluaciones subjetivas de la vida de personas de distintas edades en determinadas circunstancias (Hammond, 1995; Bakkes, 1994).

revista invi № 66 / Agosto 2009 / Volumen № 24: 89-113 
contaminación, higiene ambiental y vulnerabilidad ante catástrofes.

El campo físico, referido a equipamiento e infraestructura, comprendió un indicador denominado calidad de los servicios del barrio. Este estuvo compuesto por sub-indicadores tales como: limpieza de calles, iluminación de calles, recolección de basuras, desagües, servicio de bomberos y policías, manutención de vías públicas, postas y consultorios.

El campo financiero-económico, inherente a recursos financieros, se circunscribió a indicadores como: ingreso familiar y percepción de situación económica. Este último estuvo compuesto por los sub-indicadores: posibilidad de darse gustos, posibilidad de ahorrar, manejo del propio dinero e independencia económica.

A partir de los resultados de la encuesta, y para cada uno de losindicadoresysub-indicadores, se elaboraron índices ${ }^{7}$ de calidad de vida, que proporcionan una valoración cuantificada de los resultados. En esta oportunidad nos referiremos a los 21 indicadores, incluyendo resultados cuantitativos y cualitativos, sin llegar a desagregarlos en los 59 sub-indicadores.

Para la selección de la muestra de las áreas de la ciudad a encuestar, se partió del cruce de la

7 Entendemos por índice (www.elmundo.com), una "cifra que expresa la relación entre una serie de datos y permite sacar conclusiones". información de los mapas temáticos SIG, elaborados a partir del Censo 2002, con la sectorización de Valparaíso en Barrios ${ }^{8}$, propuesta como un instrumento operativo para la investigación. Esto permitió, coherentemente a los objetivos planteados en el proyecto, definir los barrios que cumplían con las condiciones para formar parte de la muestra. Entre los 97 barrios definidos (3 en el plan de Valparaíso y 94 en los cerros), para la muestra se buscaron aquellos que cumplieran con una densidad mínima de adultos mayores, con una diferencia socio-económica reflejada en la calidad habitacional y con una localización según cerro/ plan. De este modo, se seleccionaron trece barrios, tres en el plan: Plaza Victoria, Almendral y Sector Puerto, y diez en los siguientes cerros: Rodelillo, Polanco, Alegre Medio, Alegre Bajo, Concepción, Bellavista, Yungay, Merced Bajo, Miraflores Alto y Santa Elena. Dentro de esta muestra, se escogió como población en estudio a adultos de 60 años y más. En concreto, se encuestaron 315 adultos mayores de sexo femenino, equivalente al 65,6\% de la muestra total; y 165 adultos mayores de sexo masculino, equivalente al 34,4\%.

Para la construcción del Índice de Calidad de Vida en sí, se seleccionaron aquellas preguntas de la encuesta que apuntaban directamente hacia la calidad de vida

8 Fadda y Cortés, 2007 (pp. 56-58). 
del adulto mayor. A las respuestas se les determinó un puntaje ubicado en una escala entre 0 y 1 .

Los rangos dentro de los cuales se calificaron los índices son:

0 a 0,24 = "Muy Mala Calidad de Vida"

0,25 a 0,49 = "Mala Calidad de Vida"

0,5 a $0,74=$ "Buena Calidad de Vida"

0,75 a 1 = "Muy Buena Calidad de Vida"

Los resultados se discriminaron según sexo, edad, localización (cerro/plan), nivel socio-económico (vulnerable/estable) y zona ecológica (cerro económicamente estable, cerro económicamente vulnerable, plan económicamente estable, plan económicamente vulnerable), obteniéndose resultados para cada una de estas categorías.

Aquí haremos referencia a los resultados generales del adulto mayor en Valparaíso y a las diferencias más importantes según localización y zona ecológica.

\section{Percepción del Adulto Mayor de los Distintos Factores que Inciden en su Calidad de Vida}

Si bien la encuesta proporciona la pauta cuantitativa delanálisis, éstese hacomplementado con resultados de otros instrumentos, especialmente cualitativos, en lo, que ha constituido una triangulación.
Del análisis de los resultados más generales de la investigación, esto es, los relativos a los cinco campos en que se agruparon los indicadores de calidad de vida del adulto mayor, el económicofinanciero y el natural fueron los que obtuvieron los peores índices. El mejor, correspondió al campo humano, que se refiere a las habilidades, conocimientos, capacidades laborales y salud de los encuestados (ver Tabla 1 y Gráfico 1).

\section{TABLA 1. ÍNDICES POR CAMPO DE CALIDAD DE VIDA DEL ADULTO MAYOR}

\begin{tabular}{l|l}
\hline Campo & Índice \\
Económico-Financiero & 0,48 \\
\hline Natural & 0,48 \\
\hline Socio-Cultural & 0,52 \\
\hline Físico & 0,59 \\
\hline Humano & 0,68 \\
\hline
\end{tabular}

Fuente: elaboración propia

Pasando a profundizar, con datos más específicos, la Tabla 2 y los Gráficos 2 y 3 ilustran los campos desagregados en los 21 indicadores definidos para evaluar la calidad de vida de los adultos mayores en Valparaíso. Todos ellos muestran los Índices por indicador ordenados ascendentemente, desde los que obtuvieron la peor calificación (participación en organizaciones), hasta los mejor percibidos (uso 


\section{GRÁFICO 1. ÍNDICES POR CAMPO DE CALIDAD DE VIDA DEL ADULTO MAYOR}

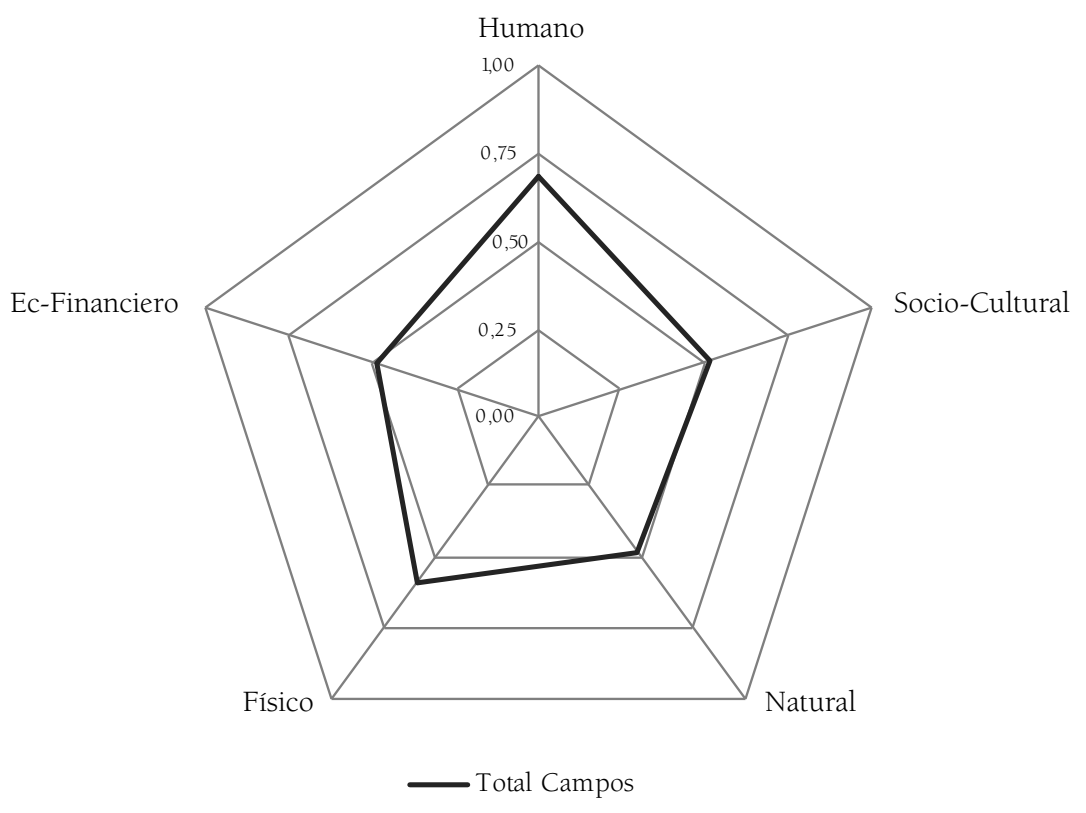

Fuente: elaboración propia

del tiempo libre). En los gráficos este orden tiene su expresión en una figura de espiral.

Mientras el Gráfico 2 expone los índices resultantes, sin discriminar por categoría, la Tabla
2 los separa por "Zona Ecológica", esto es: Cerro Económicamente Estable, Plan Económicamente Vulnerable, Cerro Económicamente Vulnerable y Plan Económicamente Estable, y el Gráfico 3 lo hace por "Localización": cerro y plan.

9 Denominación dada en el proyecto para designar zonas internamente homogéneas de acuerdo a localización y nivel socioeconómico. 
TABLA 2. ÍNDICES ORDENADOS DE MUY MALA A MUY BUENA PERCEPCIÓN

\begin{tabular}{|c|c|c|c|c|c|c|}
\hline \multirow{2}{*}{$\begin{array}{l}: \frac{\overline{0}}{0} \\
\frac{0}{0} \\
\frac{\mathrm{U}}{0} \\
0\end{array}$} & \multirow[t]{2}{*}{ Indicador } & \multicolumn{4}{|c|}{ Valor Índice según Zona Ecológica } & \multirow{2}{*}{$\begin{array}{l}\text { Valor } \\
\text { Índice } \\
\text { General }\end{array}$} \\
\hline & & $\mathrm{C}^{\circ} \mathrm{E}$ & $\mathrm{C}^{\circ} \mathrm{V}$ & $\mathrm{PIE}$ & PIV & \\
\hline \multirow{3}{*}{$\sum_{\Sigma}^{3} \frac{\pi}{2 \pi}$} & Participación en organizaciones & 0,09 & 0,06 & 0,11 & 0,08 & 0,09 \\
\hline & Necesidades del barrio & 0,23 & 0,09 & 0,19 & 0,27 & 0,20 \\
\hline & Factores de seguridad del barrio & 0,33 & 0,17 & 0,18 & 0,17 & 0,21 \\
\hline \multirow{6}{*}{$\frac{\frac{\pi}{\pi}}{\sum}$} & Contaminación & 0,33 & 0,35 & 0,22 & 0,20 & 0,28 \\
\hline & Consideración de sus opiniones & 0,52 & 0,39 & 0,35 & 0,46 & 0,43 \\
\hline & Nivel educacional & 0,57 & 0,35 & 0,44 & 0,45 & 0,45 \\
\hline & Vulnerabilidad ante catástrofes & 0,55 & 0,39 & 0,42 & 0,44 & 0,45 \\
\hline & Situación económica & 0,50 & 0,43 & 0,39 & 0,50 & 0,46 \\
\hline & Ingreso familiar & 0,57 & 0,31 & 0,47 & 0,52 & 0,47 \\
\hline \multirow{8}{*}{$\frac{\widetilde{\pi}}{\stackrel{0}{0}}$} & Calidad de servicios de su barrio & 0,61 & 0,58 & 0,55 & 0,56 & 0,57 \\
\hline & Expectativas del futuro & 0,69 & 0,56 & 0,60 & 0,59 & 0,61 \\
\hline & Estado de salud & 0,68 & 0,57 & 0,61 & 0,67 & 0,63 \\
\hline & Evaluación de su experiencia de vida & 0,72 & 0,59 & 0,68 & 0,68 & 0,66 \\
\hline & Higiene ambiental & 0,72 & 0,60 & 0,62 & 0,70 & 0,66 \\
\hline & Percepción de su barrio & 0,86 & 0,55 & 0,67 & 0,64 & 0,68 \\
\hline & Apoyo afectivo & 0,79 & 0,69 & 0,66 & 0,68 & 0,70 \\
\hline & Opinión respecto de sus vecinos & 0,82 & 0,72 & 0,75 & 0,70 & 0,75 \\
\hline \multirow{4}{*}{ 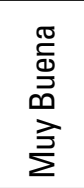 } & Discriminación en razón de la edad & 0,88 & 0,82 & 0,78 & 0,81 & 0,82 \\
\hline & Apoyo que brinda a los demás & 0,88 & 0,74 & 0,91 & 0,87 & 0,85 \\
\hline & Privacidad de la vivienda & 0,96 & 0,94 & 0,94 & 0,91 & 0,94 \\
\hline & Uso del tiempo libre & 1,00 & 0,96 & 1,00 & 0,96 & 0,98 \\
\hline
\end{tabular}

$* \mathrm{C}^{\circ} \mathrm{E}$ : Cerro Económicamente Estable $\quad *$ Pl V : Plan Económicamente Vulnerable

* $\mathrm{C}^{\circ} \mathbf{V}$ : Cerro Económicamente Vulnerable * Pl E : Plan Económicamente Estable

Fuente: elaboración propia 


\section{GRÁFICO 2. ÍNDICES POR INDICADOR ORDENADOS ASCENDENTEMENTE}

\section{Sub-Índices de Calidad de Vida del Adulto Mayor en la Ciudad de}

Valparaíso

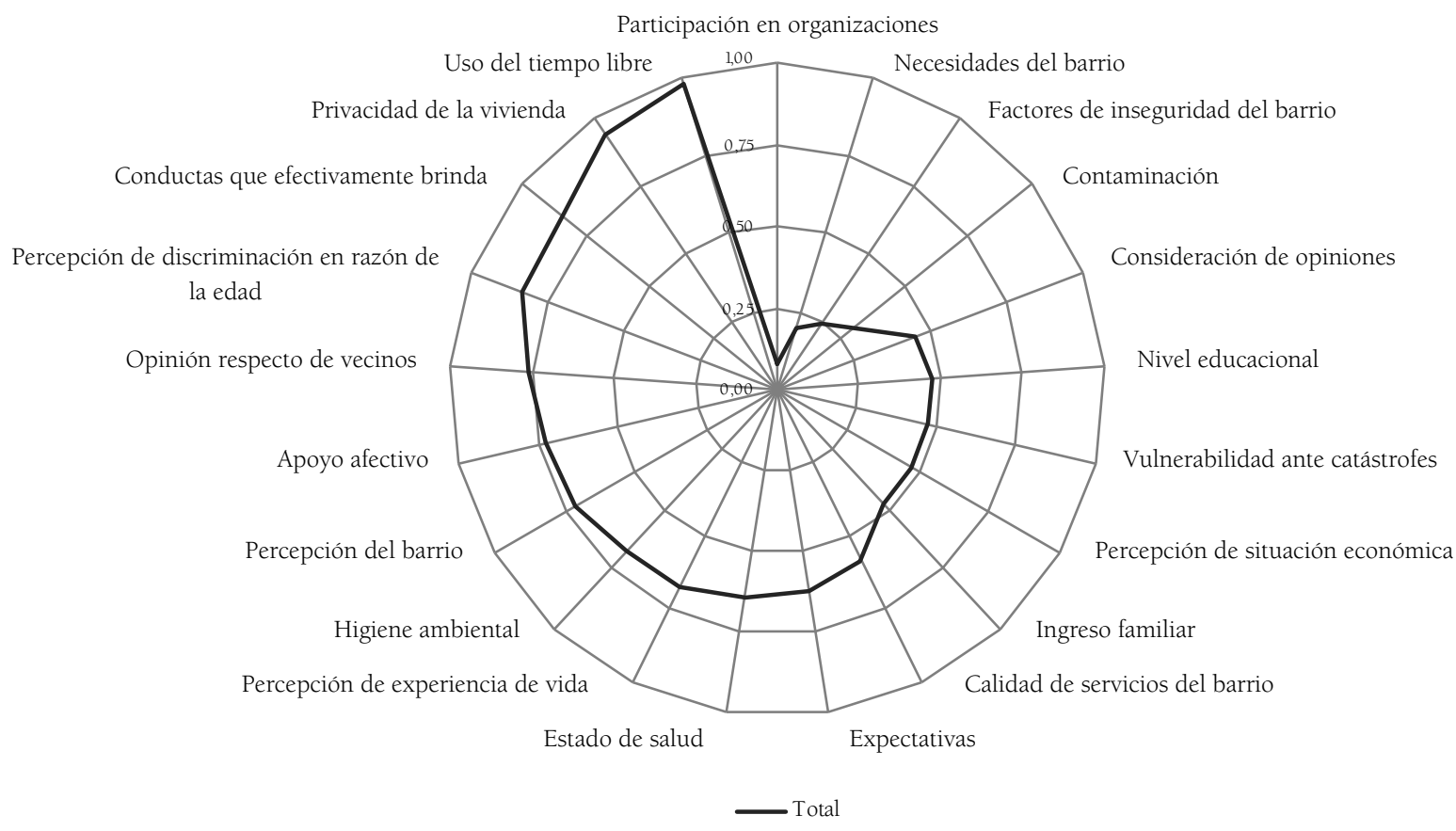

Fuente: elaboración propia 


\section{GRÁFICO 3. ÍNDICES POR INDICADOR ORDENADOS ASCENDENTEMENTE Y DISCRIMINADOS SEGÚN LOCALIZACIÓN.}

\section{Comparación de Sub-Índices de Calidad de Vida de Adultos Mayores según Localización}

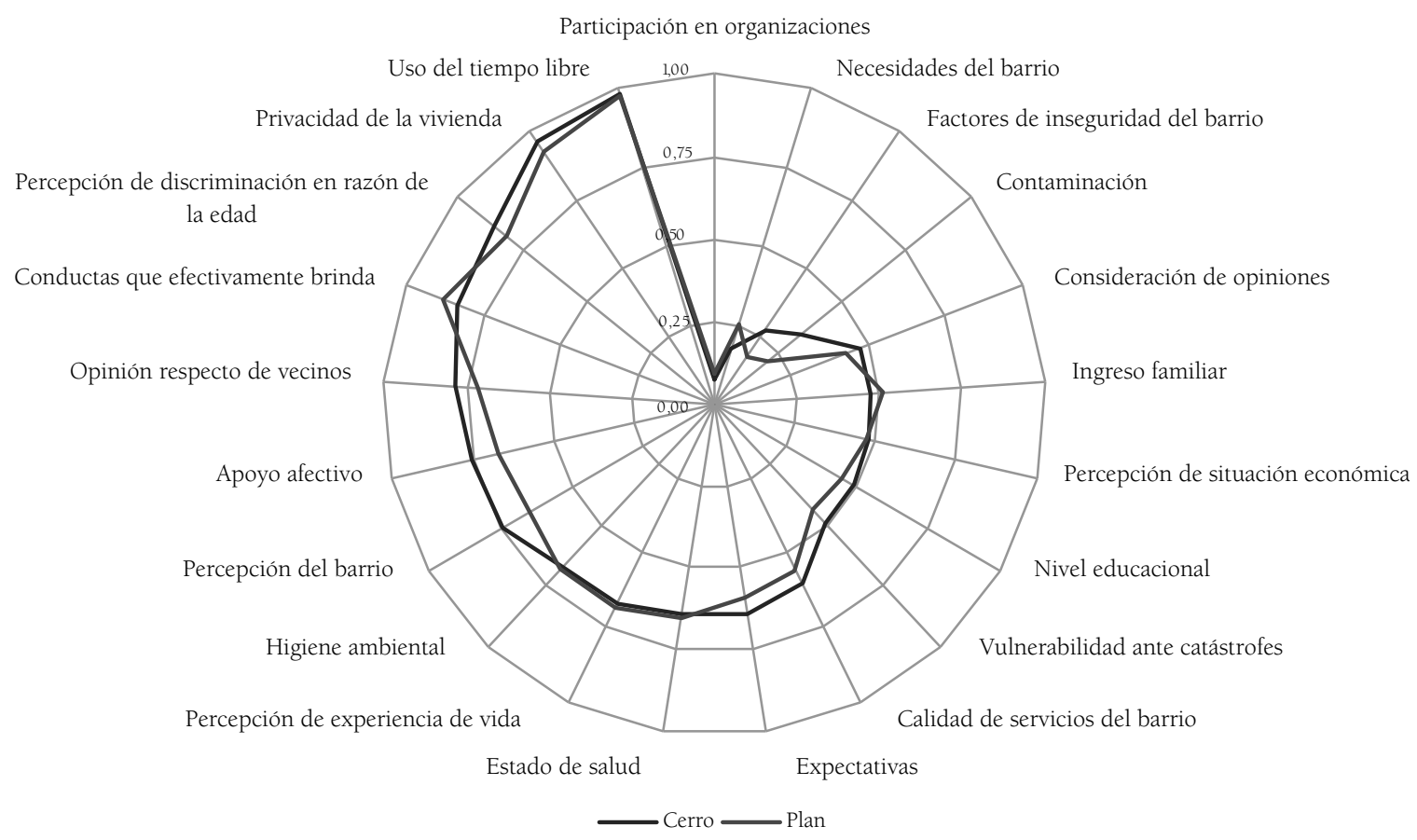

Fuente: elaboración propia 
En dichas figuras se puede apreciar que existe gran diferencia en la valoración de los distintos aspectos que concurren a la calidad de vida de los mayores en Valparaíso. Si observamos el espiral del Gráfico 2, se puede señalar que los elementos más negativamente evaluados son aquellos relacionados a la participación en organizaciones, necesidades de equipamiento y seguridad en el barrio. Los siguen en elorden ascendentelos de contaminación, falta de consideración de sus opiniones, ingreso familiar, situación económica, nivel educacional y vulnerabilidad ante catástrofes. Los índices de todos ellos se mantienen bajo la línea media de 0,5. $\mathrm{Al}$ otro extremo, percibidos como muy buenos, se ubican la no-discriminación en razón de la edad, el apoyo que el adulto mayor puede brindar a los demás, la privacidad de la vivienda y el uso de su tiempo libre. En general, se podría colegir que, a diferencia de los peor evaluados, los elementos mejor percibidos dependen más de la iniciativa particular de los adultos mayores que de intervenciones públicas.

De acuerdo a estas ilustraciones, se aprecia que cuando se discrimina por localización (cerro/plan), en general, los valores son más positivos en el cerro que en el plan (Gráfico 3). Lo que indicaría que los adultos mayores del cerro perciben una mejor calidad de vida. Los aspectos que más contribuyen a esta ventaja son: los menores factores de inseguridad en el barrio, la menor contaminación, una mejor percepción del propio barrio y un mayor apoyo afectivo.

El cerro entonces asume una dimensión positiva en contraposición al plan, visto como un espacio donde no existe vida comunitaria. La dimensión comunitaria es particularmente apreciada por los adultos mayores, ya que representa una forma para salir de la soledad sin tener que desplazarse y para "sentirse parte de". Es así como el cerro delimita el espacio donde se comparten lugares y significaciones; los sujetos se encuentran y se reúnen estableciendo relaciones permanentes. El hecho de que las personas se conozcan personalmente o se relacionen con alguien familiarmente, es un factor que hace que los adultos mayores se sientan más seguros al vivir en el cerro.

No obstante lo anterior, al agregar el factor socioeconómico (Gráfico 4 y Tabla 1), se aprecia que las ventajas del cerro no son homogéneas, sino que existe una gran diferencia entre Cerro Económicamente Estable y Cerro Económicamente Vulnerable. Ello implicaque muchas ventajas y otras tantas desventajas, se concentran en diferentes zonas, de acuerdo al nivel socioeconómico de los habitantes. Por ende, se constata una segregación en los cerros, los cuales albergan al mayor porcentaje de habitantes.

Mientras en el plan no se presentan grandes diferencias, en los cerros podemos apreciar enormes brechas según zonas socio-económicas. Por ejemplo, 


\section{GRÁFICO 4. ÍNDICES POR INDICADOR ORDENADOS ASCENDENTEMENTE Y DISCRIMINADOS SEGÚN ZONA ECOLÓGICA.}

\section{Comparación de Sub-Índices de Calidad de Vida de Adultos Mayores}

según Zona Ecológica

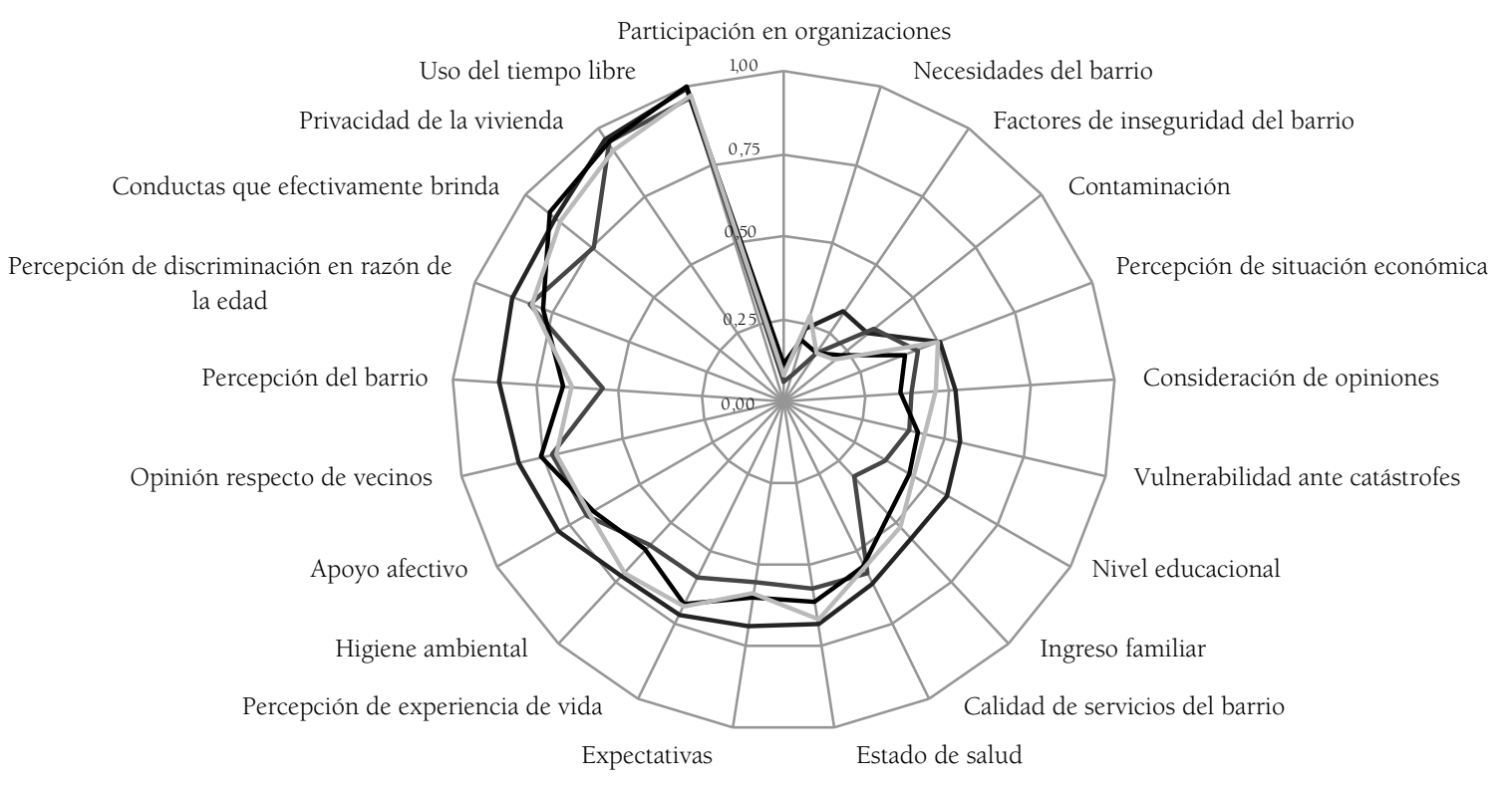

_ Cerro Económicamente Estable

Cerro Económicamente Vulnerable

—Plan Económicamente Estable _Plan Económicamente Vulnerable

Fuente: elaboración propia 
el aspecto de la percepción del barrio es el que arroja la mayor diferencia entre la evaluación que hacen los habitantes de una u otra zona. Ello implica un gran desarraigo y falta de identidad en los barrios más pobres, no así en los más estables. Otros factores percibidos también con gran desigualdad en las dos zonas de los cerros son los relativos a la situación económica, el nivel educacional, la inseguridad en el barrio, la vulnerabilidad ante catástrofes y la falta de consideración de sus opiniones.

Se constata, entonces, una gran segregación dentro de los cerros, segregación que resulta más dura para los ancianos. Ella, de acuerdo a las propias manifestaciones de los encuestados, incide sensiblemente en su calidad de vida, especialmente en los grupos más vulnerables.

A continuación se analizan los indicadores que incidieron más determinantemente -negativa o positivamente- en la calidad de vida de los adultos mayores en Valparaíso. De acuerdo a lo anterior, ellos se agruparon en dos categorías: Indicadores con Incidencia Negativa e Indicadores con Incidencia Positiva.

\section{INDICADORES CON INCIDENCIA NEGATIVA}

\section{Participación en Organizaciones (índice 0,09).}

Este indicador se midió a partir de la participación del adulto mayor en Juntas de vecinos, Centros de madres, Partidos políticos, Clubes de adultos mayores, Grupos religiosos, Clubes deportivos, Fiestas de barrio, Reuniones sociales, Grupos de música o baile y otras actividades. De todas ellas, donde más participan los adultos mayores son en grupos religiosos y en reuniones sociales.

En los instrumentos cualitativos fue muy recurrente que en los relatos de las personas surgiera la referencia a la dimensión religiosa. En algunos sujetos la condición de su edad actual es experimentada como un estado de gracia divina, en otros las atribuciones religiosas permiten entregarse con serenidad a los acontecimientos presentes y futuros. La fe en muchos casos es un recurso para sobrellevar los problemas que se presentan, para enfrentar esta etapa de manera positiva. En particular, el entregarse a Dios representa un recurso para las personas que viven solas y que no cuentan con un apoyo directo en caso de necesidad.

Al discriminar el indicador Participación en Organizaciones por nivel socioeconómico, son quienes viven en las áreas Económicamente Vulnerables, tanto en el cerro como en el plan, quienes menos participan en organizaciones. Esto es de especial preocupación si se toma en cuenta que estas actividades pueden fomentar la creación de redes sociales y de colaboración mutua, que permitan enfrentar de mejor manera la marginalidad socioeconómica.

En los testimonios rescatados, las vivencias de soledad que los adultos mayores experimentan son 
componentes clave para describir sus percepciones en torno a las redes sociales; aquellas vivencias dejan entrever las razones por las cuales los sujetos se insertan en espacios de participación que facilitan el encuentro con otros y con esto permiten asumir de mejor forma su actual situación.

\section{Necesidades del Barkio y Factores de Seguridad del Barrio (ÍNdices 0,20 y 0,21 Respectivamente).}

Las necesidades del barrio fueron evaluadas a partir del equipamiento urbano: locomoción, áreas verdes, clubes y equipamiento de salud, deportivo, cultural y comercial. Fueron los residentes de los cerros económicamente vulnerables quienes tuvieron una peor percepción, especialmente entorno a locomoción, áreas verdes y plazas.

En cuanto a los factores de Seguridad del barrio, la única zona que presenta una percepción más positiva es el cerro económicamente estable. Los principales factores que causan inseguridad en el barrio que habita el adulto mayor son la delincuencia, las drogas, el alcoholismo y la falta de iluminación, observándose una percepción más negativa en el plan que en el cerro, diferencia que se hace más notoria con respecto al cerro económicamente estable.

Los instrumentos cualitativos hacen referencia al deterioro de las vías públicas y su consecuente obstaculización al desplazamiento. El deterioro urbano, especialmente la mala calidad de las calles, es el aspecto que los adultos mayores identifican como más problemático, en cuanto que pone en riesgo la indemnidad física de la persona al transitar en el espacio urbano. Los adultos mayores que viven en el cerro bajan constantemente al plan para realizar sus trámites, acudir a los servicios o simplemente para ahorrar en las compras domésticas.

En los relatos de los adultos mayores emerge transversalmente la sensación de inseguridad que experimentan en el espacio público, así como en el espacio doméstico. Se reconocen como un grupo vulnerable desde esta perspectiva, y blanco fácil para la microcriminalidad urbana. Precisamente, esta sensación de inseguridad y vulnerabilidad condiciona el actuar de los adultos mayores, determinando el uso de ciertos espacios urbanos y preferentemente en ciertos horarios, llegando en casos extremos a transformar la percepción del propio barrio y fomentando comportamientos de desconfianza y auto segregación.

\section{Contaminación (ÍNdice 0,28).}

Los principales factores de contaminación por los que se sienten mayormente afectados los adultos mayores son: la basura, los ruidos, los animales y el smog (calidad del aire). Los resultados permiten distinguir que el plan es percibido como una zona más contaminada que los cerros. El plan concentra peores evaluaciones en torno a los mencionados cuatro factores, mientras que los cerros aparecen 
más afectados por los animales callejeros, insectos $y$, en menor grado, por problemas de desagües y olores.

Se advierte que la contaminación por basura y calidad del aire se da mayoritariamente en zonas económicamente vulnerables. En cambio, la contaminación acústica y el problema de los animales e insectos tienen mayor frecuencia en zonas económicamente estables.

Los resultados obtenidos a través de los instrumentos cualitativos, sugieren que el cerro es el lugar privilegiado para vivir alejado de la contaminación. Si bien, ningún lugar se ve exento a este problema, el cerro es considerado un espacio predilecto, no sólo por las vistas panorámicas, sino también por su calidad del aire, y en general por una menor contaminación acústica. En este sentido, la vida en el cerro para el adulto mayor presenta, según sus discursos, una visión más saludable que la del plan. Ellos critican la responsabilidad de las autoridades locales, en el mantenimiento de los espacios públicos, basura e higiene ambiental en general.

\section{Falta de Consideración de sus Opiniones (índice 0,43).}

Al analizar si el adulto mayor siente que sus opiniones son consideradas en distintas escalas de participación, como son las municipalidades, las organizaciones comunitarias, su barrio y su vivienda, el $73,5 \%$ de ellos percibe que sus opiniones son bastante consideradas en la vivienda que habita. El $47,5 \%$ señala que sus opiniones no son nada consideradas en el barrio, percepción que resulta aún más negativa en la zona económicamente vulnerable. El 57,5\% señala que sus opiniones no son atendidas por las organizaciones comunitarias, habiendo menor participación en los cerros que en el plan, en dichas organizaciones. Por otra parte, la mayor cantidad de adultos mayores que no participa en organizaciones comunitarias se ubica en zonas económicamente estables. En cuanto a la receptividad por parte de la autoridad local, un $53,5 \%$ de los encuestados señala que sus opiniones no son escuchadas en absoluto por la Municipalidad.

Los testimonios recopilados denotan que los adultos mayores empiezan a reconocerse como sujetos con derechos, contraponiéndose de esta forma a la tradicional exclusión de las medidas político-sociales y de los relativos beneficios que de éstas pueden derivar. Se trata de un autoreconocimiento, todavía en germen, fomentado principalmente por la relevancia que se le da al tema del adulto mayor a nivel nacional y en menor medida por la generación de políticas que buscan mejorar las condiciones de envejecimiento.

\section{Nivel educacional (índice 0,45).}

El 24,6\% de los encuestados tiene educación básica incompleta. El 16,9\% media completa y sólo el 6,3\% 
tiene educación universitaria completa. Se observa que aquéllos que realizaron estudios universitarios y enseñanza técnica, ya sea de manera íntegra o parcial, se ubican preferentemente en los cerros económicamente estables. En cambio, el 52,1\% de quienes viven en los cerros económicamente vulnerables tienen educación básica incompleta.

\section{Vulnerabilidad ante Catástrofes (índice de 0,45).}

Al consultar a los adultos mayores si se sentían amenazados por alguna catástrofe natural (temporales, temblores, aluviones, derrumbes, inundaciones y desbordes), ellos manifestaron sentirse amenazados principalmente por temblores y temporales. Se observa que la percepción respecto a los temblores es similar para todas las zonas: alrededor de un $40 \%$ se siente amenazado por este tipo de fenómeno. En relación a los temporales, quienes viven en el cerro económicamente vulnerable se sienten más amenazados. Por el contrario, quienes viven en el cerro económicamente estable son los que menos amenazados se sienten frente a estos posibles desastres.

La información cualitativa refleja que, frente a un tsunami, existe mayor percepción de peligro por parte de quienes viven en el plan. Vivir en el cerro se percibe como más seguro frente a catástrofes asociadas al mar.

\section{Percepción de la Situación Socioeconómica e Ingreso Familiar (Índice de 0,46 y 0,47 respectivamente).}

La media del ingreso familiar de los encuestados fue de $\$ 278.431$ y la del ingreso recibido por el adulto mayor fue de $\$ 159.562$.

La percepción que los adultos mayores tienen respecto de su situación económica se evaluó a partir de la posibilidad de darse gustos, la posibilidad de ahorrar, el manejo de su propio dinero y su independencia económica, antes y después de haber jubilado o sencillamente de antes y después de haber cumplido los 60 años.

Por ejemplo, en relación a la posibilidad de darse gustos, una vez jubilado o cumplido los 60 años, un $36 \%$ aproximadamente considera que su situación empeoró, y un 43,3\% que sigue igual de bien. La tendencia es que empeoró mayoritariamente en el cerro económicamente vulnerable y la situación sigue igual de bien principalmente en el cerro económicamente estable.

Los instrumentos cualitativos dan cuenta de realidades extremas: la jubilación no alcanzaría para cubrir los gastos familiares o personales. En algunos casos, si bien las personas tienen solventadas sus necesidades básicas, remarcan el hecho de que la pensión no les permite acceder a mejor calidad de vida puesto que no pueden satisfacer necesidades 
de corte recreativo y ven restringidos el acceso a espacios de socialización.

\section{INDICADORES CON INCIDENCIA POSITIVA}

\section{Calidad de los Servicios del Barrio (índice 0,57).}

Este indicador se refiere al equipamiento relacionado con: iluminación, servicio de recolección de basura, policía, bomberos, mantenimiento de vías públicas, posta y consultorio.

El $66,9 \%$ de los encuestados considera que la iluminación es buena o muy buena, siendo evaluada en forma menos positiva en el plan económicamente estable y las mejores opiniones se ubican preferentemente en el cerro económicamente vulnerable. En cuanto a la recolección de basura, el 83,6\% la considera buena o muy buena, siendo los habitantes del cerro, quienes tienen una visión más positiva. Por otra parte, el $82,2 \%$ de la muestra considera bueno o muy bueno el servicio de bomberos, lo que es especialmente válido en el cerro económicamente estable. Similarmente, el $75 \%$ considera el servicio policial como bueno o muy bueno. La opinión sobre la calidad del servicio de mantenimiento de las vías públicas está dividida: 50,4\% lo califica positivamente (buena y muy buena) y un 49,2\%, en forma negativa (muy mala y mala), siendo más negativa en el plan que en el cerro. A través de los instrumentos cualitativos, ésta resultó negativa, no así el servicio de salud, que fue calificado muy positivamente, incluso expresando sentimientos de gratitud y bienestar. En la encuesta este sub-indicador obtuvo un índice de 0,61, subiendo la media del indicador Calidad de los Servicios del Barrio.

Dentro de los servicios públicos que ofrece el aparato gubernamental, los consultorios y centros de salud se encuentran entre los más demandados por los adultos mayores. Uno de los aspectos que los entrevistados revelan como muy importante, y que contribuye a su percepción positiva, es el beneficio de la gratuidad tanto de la atención médica, como de los medicamentos, entrega de alimentos y constancia en los controles periódicos, realizados por médicos y enfermeras. También es valorizado el apoyo psicológico implementado para ayudar al adulto mayor a enfrentar y superar estados de aislamiento psico-social.

En relación al Estado de Salud, según el análisis estadístico, un $64,6 \%$ de los adultos mayores se ve afectado por algún tipo de enfermedad física, siendo esto más frecuente en la mujer que en el hombre y en zonas económicamente vulnerables que en las estables. Los chequeos médicos suelen hacerse más frecuentemente preventivamente que frente a una enfermedad ya declarada.

Otros tipos de apoyo son los programas y proyectos brindados por las instancias municipales. Los entrevistados los asocian a experiencias agradables, 
valorizando la posibilidad que se les brinda de participarenactividades recreativasydecapacitación. Los testimonios de los grupos focales reflejan un adulto mayor informado, activo, participante de las redes ofrecidas por el gobierno local.

El imaginario negativo de los servicios públicos se cristaliza en torno a la percepción acerca de las casas de acogida, a pesar de que ninguno de los sujetos consultados es usuario directo. La existencia de estos espacios representa, para ellos, la situación del abandono de la tercera edad, por lo tanto, le asignan atributos negativos. Esta visión resulta a partir del conocimiento de experiencias ajenas, difundidas por los medios de comunicación.

\section{Expectativas del Futuro (índice 0,61).}

El 53,6\% de los encuestados señala que ve su futuro como bueno o muy bueno, produciéndose en el cerro económicamente estable, la visión más positiva. La información cualitativa proporciona un panorama más diversificado, donde aparecen expectativas tanto positivas como negativas.

Frente a la percepción de su propio envejecimiento, en general, los adultos mayores poseen una percepción positiva. Sin embargo, en algunos relatos emerge una connotación negativa de esta etapa de vida como resultado de consideraciones sobre el decaimiento físico y de las condiciones económicas, factores vivenciados como limitantes de las posibilidades de vivir dignamente y disfrutar de la vida.
Entre las percepciones positivas de esta etapa hay sujetos que resaltan el hecho de haber logrado superar las dificultades de la vida, dando un nuevo significado a su experiencia vital y valorizando su situación actual. El hecho de aceptar positivamente su condición etaria los lleva a manifestar un fuerte apego a la vida. El asumir la edad con espíritu positivo es vivenciado como factor que permite desarrollar actividades y disfrutar de lo que ofrece la vida. Muchas personas enfrentan con optimismo esta etapa.

\section{Experiencia de Vida hasta Ahora (índice 0,66).}

Según el análisis estadístico, la percepción de los adultos mayores en torno a su experiencia de vida hasta ahora, en general, es positiva. Un $57,7 \%$ encuentra que es buena y un $10 \%$ la considera muy buena. Sólo alrededor de un 5\% considera que su experiencia de vida ha sido mala o muy mala, y un $25 \%$ la considera como regular. La capacidad de haber superado en sus trayectorias personales ciertas dificultades, generaría un sentimiento de satisfacción por su condición actual. La evaluación de la experiencia de vida resultó más negativa (muy mala, mala y regular) en las zonas económicamente vulnerables. Por el contrario, las evaluaciones positivas (buena y muy buena) se concentraron más en zonas económicamente estables. Los resultados obtenidos, mediante los instrumentos cualitativos, confirman la evaluación positiva general. 


\section{Higiene Ambiental (índice 0,66).}

Este indicador está compuesto por elementos como la existencia de microbasurales y plagas. Si bien su evaluación es positiva, ésta se ve afectada por factores negativos como la presencia de los perros callejeros, ratones y palomas. El 53,8\% de los encuestados se siente perturbado por el problema de los perros callejeros de Valparaíso, el 40,6\% por las palomas y el $32,9 \%$ por los ratones. Dentro de los métodos cualitativos, aparece especialmente mencionado el tema de los perros callejeros y se lo asocia directamente a la contaminación por basura.

\section{Percepción de su Barrio (índice 0,68).}

Este indicador se refiere a si el barrio es percibido como pobre o no pobre, feo o bonito, ideal para vivir o no ideal para vivir, seguro o inseguro y si le gustaría cambiarse de él. Un 67,3\% considera su barrio como no pobre. Alrededor de un $75 \%$ lo considera un lugar bonito, un $75 \%$ lo considera ideal para vivir, alrededor de un 65\% lo considera un lugar seguro, alrededor de 57\% no estaría dispuesto a cambiarse. Como puede apreciarse, existe una percepción bastante buena del hábitat inmediato, lo cual incide en una buena identificación y arraigo de las personas con su entorno. Al discriminar por nivel socioeconómico, se puede concluir que todos estos factores son percibidos de manera más negativa por los habitantes de las zonas más vulnerables.
De los instrumentos cualitativos se desprende que existe en general un gran arraigo al barrio donde se habita. Esto es más fuerte en los cerros, donde existe más vida comunitaria y carga simbólica.

\section{Apoyo Afectivo (índice 0,70).}

Al analizar este indicador se detecta que el 19,6\% de los adultos mayores encuestados vive solo, que un $66 \%$ se siente muy apoyado por sus hijos y el $53,9 \%$, muy apoyado por sus nietos. Un 34,6\% de los encuestados se siente muy apoyado por su pareja, mientras que un $10 \%$ afirma no recibir tal apoyo (49,8\% no tiene pareja). Un $47 \%$ que se siente muy apoyado por los amigos. El $40 \%$ de los adultos mayores se siente muy apoyado por los vecinos, observándose, también en este aspecto, una mayor frecuencia en el cerro. El apoyo afectivo por parte de los vecinos y amigos fue mayormente percibido en las zonas económicamente estables.

Deacuerdoalosdatosrecopiladosporlosinstrumentos cualitativos, hay adultos mayores que sienten una ausencia de apoyo afectivo principalmente por parte de la familia. En tales casos, los grupos de amigos y vecinos resultaron de gran apoyo.

La soledad induce a los adultos mayores a la creación de mecanismos para suplir la falta de vínculos significativos. Para ello, predomina la estrategia de construcción e inserción en redes sociales formales e informales a fin de establecer relaciones de apoyo mutuo, solidaridad y compañía. Se crea, 
de esta forma, un mecanismo de intercambio recíproco, que permite enfrentar la vida con más seguridad. El apoyo social que reciben de estas redes se complementa mutuamente y contribuye a satisfacer en forma global las necesidades de los adultos mayores. El tipo de apoyo que reciben varía desde la creación de espacios de socialización y participación, hasta la satisfacción de necesidades básicas, como pueden ser las relacionadas con el cuidado de la salud. Para quienes experimentan la vejez en una situación de total soledad, dichas redes constituyen un soporte fundamental, en cuanto únicas fuentes de apoyo psicosocial.

\section{Opinión de sus Vecinos (índice 0,75).}

El 84\% de los encuestados opina que sus vecinos no son conflictivos. A medida que aumenta la edad, la opinión en torno a los vecinos como agentes conflictivos disminuye. Según localización a la que pertenece el adulto mayor, se puede verificar que no hay grandes diferencias entre cerro y plan, en cambio sí la hay según zonas socio-económicas: la opinión que considera a los vecinos como conflictivos es mayor en la zona económicamente vulnerable. Por otra parte, un $70,8 \%$ de los adultos mayores considera que los vecinos son amistosos. Esto es más frecuente entre los adultos mayores que viven en el cerro y en la zona económicamente estable. Adicionalmente, el 68,3\% de los adultos mayores considera solidarios a sus vecinos. También en este caso, esto sucede con mayor frecuencia en el cerro y en la zona económicamente estable.
Los cuatro indicadores siguientes fueron los mejor calificados, con Muy Buena Percepción:

\section{Discriminación en Razón de la Edad (índice 0,82).}

Un $83,8 \%$ de los entrevistados no se ha sentido discriminado por su edad. No obstante, hay algunos aspectos, como el poco respeto, la falta de trabajo y la falta de atención en general y en especial en el transporte, que inciden negativamente en este indicador.

\section{Apoyo que Brinda a los Demás (índice 0,85).}

Entre las principales conductas con que el adulto mayor dice apoyar a los demás se destacan: la experiencia que les permite dar buenos consejos o sugerencias $(31,5 \%)$, la experiencia que les permite hacer mejor las cosas $(22,3 \%)$ y la compañía y afecto $(20,4 \%)$.

En la zona económicamente vulnerable prevalecen la experiencia ligada a hacer mejor las cosas, la importancia de la compañía y el afecto. En cambio, en la zona económicamente estable prevalece la experiencia ligada a dar buenos consejos.

\section{Privacidad en la Vivienda (índice 0,94).}

El análisis estadístico indica que el 53,1\% de los encuestados es propietario y el 33,6\%, arrendatario de la vivienda que habita. El resto de ellos son allegados, copropietarios, coarrendatarios o de vivienda cedida. Por otra parte, un 94,4\% considera que su vivienda ofrece la privacidad necesaria. 
Uso del Tiempo Libre (índice 0,98).

Este indicador fue el mejor evaluado y hace mención a las actividades que realiza el adulto mayor para entretenerse. Entre las principales figuran: ver televisión (19,2\%), realizar trabajos manuales $(15,5 \%)$, tareas relacionadas con el hogar (14\%), salir a caminar $(10,4 \%)$ y leer $(8,5 \%)$.

\section{PERCEPCIÓN GENERAL}

Cerrando la encuesta, se hicieron preguntas generales para medir si Valparaíso es o no percibida como una ciudad amable con las personas de edad y qué calidad de vida les proporciona. El 78,3\% de los encuestados considera que la ciudad es amable para vivir.

El gráfico 5 ilustra que la amabilidad de la ciudad con las personas mayores es Muy Buena, mientras que la Calidad de Vida no alcanza a llegar a la media. Al discriminar por Zonas Ecológicas (Gráfico 6) existen amplias brechas entre zonas estables y vulnerables, siendo el cerro económicamente estable el que presenta la evaluación más positiva en ambos aspectos.

\section{GRÁFICO 5. ÍNDICE DE PERCEPCIÓN GENERAL}

ÍNDICE POR PERCEPCIÓN GENERAL

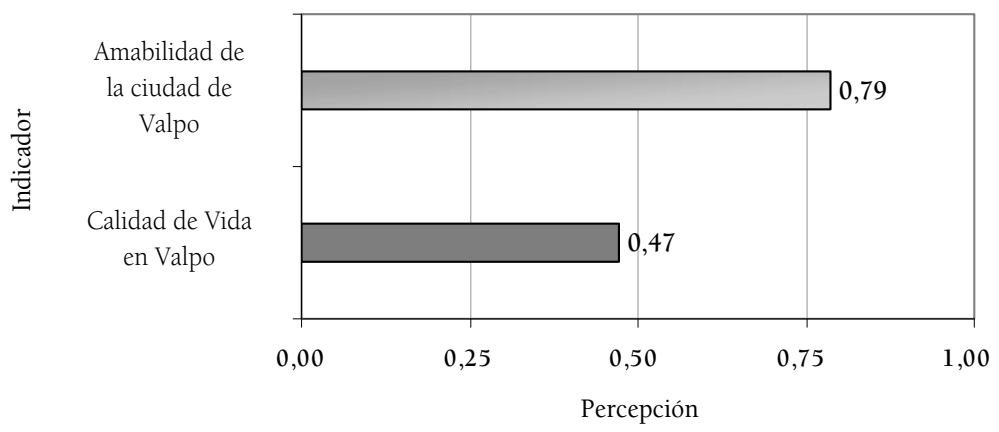

Fuente: elaboración propia 


\section{GRÁFICO 6. ÍNDICE DE PERCEPCIÓN GENERAL DISCRIMINANDO POR ZONA ECOLÓGICA}

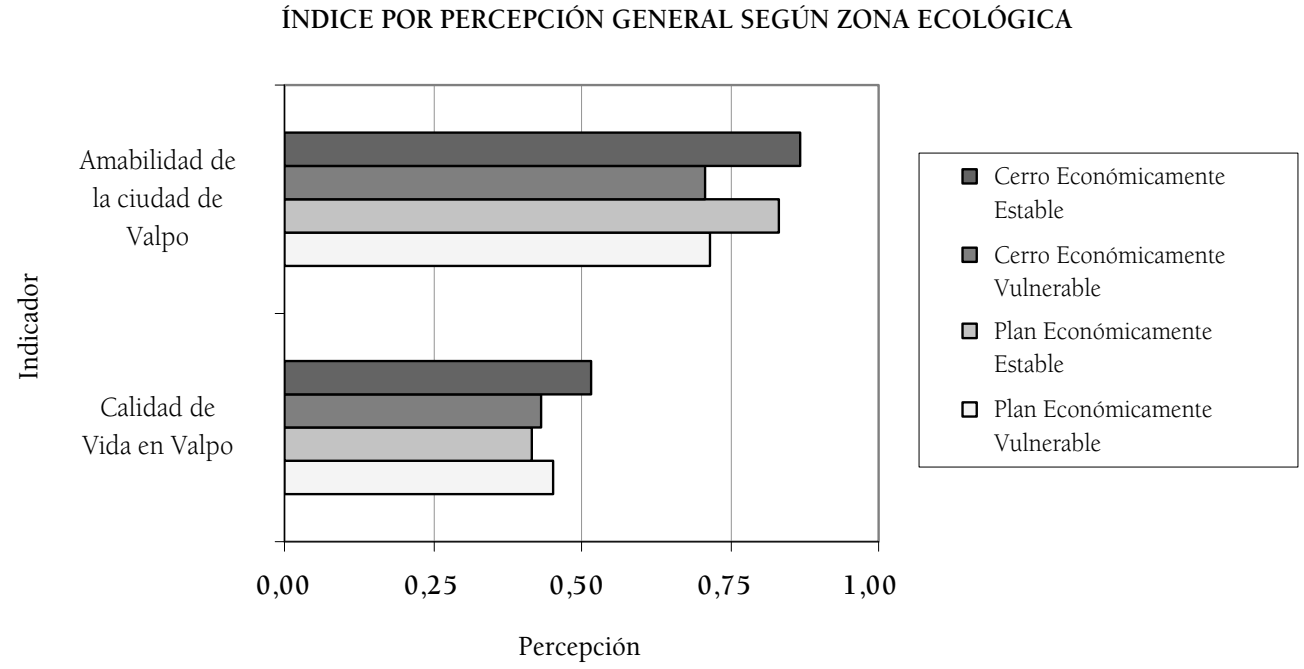

Fuente: elaboración propia

\section{Conclusiones}

A pesar de las dificultades que los adultos mayores experimentan para desplazarse entre el cerro y el plan, comúnmente, las personas le asignan un valor positivo al habitar en el cerro. Esta percepción estaría dada por un conjunto de atributos que hacen del cerro un espacio más amigable, más saludable, donde la dimensión comunitaria favorece la construcción de vínculos y redes de apoyo. Vivir en el cerro se constituye en un inductor de la identidad social; las personas se reconocen y reafirman a través de su barrio. Es así que el cerro delimita el espacio donde se comparten lugares y significaciones; los sujetos se encuentran y se reúnen estableciendo relaciones permanentes.

A pesar que las condiciones topográficas y el deterioro de las calles de Valparaíso podrían sugerir que la ciudad es dificultosa y poco amigable con las 
personas deedad, los resultados de esta investigación no indican lo mismo. Además, del análisis se puede deducir que los atributos favorables y desfavorables percibidos por los adultos mayores, están dando una pauta sobre las cosas a emular o enmendar en el desarrollo de la ciudad.

Al comparar los elementos positivos con los negativos, llama la atención que estos últimos son, mayoritariamente, aspectos de competencia de las autoridades, mientras que los positivos son más bien atribuibles a la responsabilidad propia de los afectados. Hecho que resulta coherente con la mejor calificación obtenida por el Campo Humano. Estos resultados están, por una parte, indicando que los adultos mayores tienen una actitud proactiva frente a su condición, en contraste con el sector público, que, salvo algunas excepciones, no haimplementado políticas, locales y nacionales, para enfrentar los problemas de una sociedad en progresivo envejecimiento. Por otro lado, los resultados están evidenciando cuáles son las áreas más frágiles, gran parte de las cuales son de responsabilidad pública, y susceptibles de mejorar a través de una buena gestión y planificación del desarrollo urbano en aras de una mejor calidad de vida urbana para el adulto mayor. Esas áreas debieran ser abordadas por las autoridades correspondientes para su perfeccionamiento.

Si se pretende mejorar la calidad de vida del adulto mayor, los indicadores que resultaron peor evaluados (participación en organizaciones, equipamiento del barrio y seguridad del barrio) deben ser considerados como áreas prioritarias a abordar en las políticas públicas locales, por ejemplo: estimulando la participación de este grupo etario en organizaciones y así, fomentar y reforzar sus redes sociales; haciendo inversiones en equipamiento urbano, especialmente en un sistema eficiente de transporte público que considere y tome medidas adecuadas a las limitaciones físicas y económicas del grupo, en un plan de desarrollo de áreas verdes, sobre todo en las partes más desatendidas de los cerros y en programas y/o centros deportivos y culturales especialmente dirigidos a estos sectores; e implementando estrategias para mejorar la seguridad ciudadana, especialmente en espacios públicos de alta concurrencia de este grupo etario.

Para potenciar una sinergia entre la participación comunitaria y los déficit detectados, podría pensarse en un plan de acción entre autoridades y este grupo etario. Cabe resaltar que la consideración de sus opiniones, especialmente las relacionadas a la Municipalidad, fueron mal evaluadas. Si se busca integrar socialmente a este grupo etario, es primordial mejorar este aspecto dándole posibilidad de acceso a la toma de decisiones.

Otro aspecto a considerar es el bajo nivel educacional detectado en la población adulta mayor 
de Valparaíso. Problema que supera el ámbito local y debe ser abordado con políticas públicas a nivel nacional. Esto se relaciona con otros aspectos críticos como la situación económica y las bajas jubilaciones, también detectadas como perjudiciales en su calidad de vida. Si bien la condición de jubilado proporciona ciertas gratificaciones, como contar con mayor tiempo libre y menores responsabilidades, el aspecto económico impone muchas privaciones, hasta para desplazarse en la ciudad.

Dentro de los servicios urbanos mejor evaluados, se encuentran los de salud. Los adultos mayores reconocen una atención focalizada hacia ellos mediante los programas de salud pública. Esto estaría indicando un logro de este tipo de políticas por parte del Estado, lo cual es un ejemplo a ser replicado en otras áreas, entre ellas la educación y el sistema previsional.

Por último, hay toda una visión positiva dentro de las percepciones de los adultos mayores y que tienen que ver con sus expectativas de futuro, su evaluación de la experiencia de vida, su apoyo afectivo, discriminación en razón de la edad y percepción del barrio. Hipotéticamente, todo esto podría relacionarse, de alguna forma, a su relación de arraigo con el barrio y la ciudad y a la amabilidad que ésta les ofrece. Hecho que estaría indicando que el hábitat urbano de Valparaíso proporciona condiciones propicias para la calidad de vida de este grupo de población.

\section{Bibliografía}

ÁLVAREZ, LUIS. Origen de los Espacios Públicos en Valparaíso: Discurso Higienista y las Condiciones Ambientales en el Siglo XIX. En Revista de Urbanismo No4, Santiago, ISSN 0717-5051, Ed. D. Urbanismo, F.A.U. - SISIB, U. Chile. En línea. 2001. [fecha de consulta: 23 marzo 2009]. Disponible en http:// revistaurbanismo.uchile.cl/n4/alvarez/alvarez.htm

BAKKES, J. Et al. An Overview of Environmental Indicators: State of the art and perspectives. En RIVM. UNEP, Nairobi Environmental Assessment Sub-Programme, United Nations Environment Programme, 1994. 63 p.

FADDA, GIULIETTA Y CORTÉS, ALEJANDRA. Barrios. En busca de su definición en Valparaíso. En URBANO. Noviembre de 2007 Nº 16, págs. 50-59.

FADDA, GIULIETTA Y JIRON, PAOLA. Calidad de Vida: una Metodología para la Investigación Urbana. En Revista de la Escuela de Economía y Negocios. Marzo 1999 Nº 1, págs. 175-188.

HAMMOND, ALLEN. Et al. Environmental Indicators: A Systematic Approach to Measuring and Reporting on Environmental Policy Performance in the Context of Sustainable Development. Washington D.C. World Resources Institute, 1995. 58 p.

WAISBERG, MYRIAM. Valparaíso: el legado urbanístico y arquitectónico. En Revista de la Facultad de Arquitectura. Vol 3, № 3, págs 5-9.

EL MUNDO. En línea 2005. [fecha de consulta: 25 marzo 2009]. Disponible en: www.elmundo.com

revista invi № 66 / Agosto 2009 / Volumen № 24: 89-113 113 